\title{
The Chillingworth class is a signed stable length
}

\author{
INGRID IRMER
}

\begin{abstract}
An orientation is defined on a family of curve graphs on which the Torelli group acts. It is shown that the resulting signed stable length of an element of the Torelli group is a cohomology class. This cohomology class is half the dual of the contraction of the Johnson homomorphism, the so-called "Chillingworth class".
\end{abstract}

$20 \mathrm{~J} 05 ; 47 \mathrm{~B} 47$

\section{Introduction}

Let $S_{g}$ be a closed, oriented surface with genus $g \geq 3$, and $S_{g, 1}$ an oriented surface with genus $g \geq 3$ and one boundary curve. The mapping class group of $S_{g}$, denoted $\operatorname{Mod}\left(S_{g}\right)$, is the group of isotopy classes of orientation preserving homeomorphisms of $S_{g}$. The group $\operatorname{Mod}\left(S_{g, 1}\right)$ is defined similarly, with the added condition that the homeomorphisms act as the identity on the boundary of $S_{g, 1}$. The $\operatorname{group} \operatorname{Mod}\left(S_{g}\right)$ induces an action on $H_{1}\left(S_{g}, \mathbb{Z}\right)$, and the Torelli group $\mathcal{T}_{g}$ is the kernel of this action. The Torelli group $\mathcal{T}_{g, 1}$ is similarly defined.

Johnson homomorphisms The Johnson homomorphisms (see Johnson [10]) are homomorphisms

$$
t_{g, 1}: \mathcal{T}_{g, 1} \rightarrow \Lambda^{3} H_{1}\left(S_{g, 1} ; \mathbb{Z}\right) \quad \text { and } \quad t_{g}: \mathcal{T}_{g} \rightarrow \Lambda^{3} H_{1}\left(S_{g} ; \mathbb{Z}\right) / H_{1}\left(S_{g} ; \mathbb{Z}\right) .
$$

The Johnson homomorphisms are among the most basic structures in the study of the Torelli group, for example for understanding group homology (see Johnson [11]) and 3-manifold theory (see Morita [13]). Chillingworth [5] used a notion of winding number to define the Chillingworth class, a map from the Torelli group to an integral first cohomology class of $S_{g, 1}$ or $S_{g}$. It was shown that the vanishing of this map is a necessary condition for a mapping class in the Torelli group to be in the kernel of the Johnson homomorphism, ie the Johnson kernel. Johnson [10, Theorem 2] showed that the Chillingworth class is dual to a contraction of the Johnson homomorphism. For surveys on the Johnson homomorphism, see Johnson [11] and Farb and Margalit [7]. 
Curve graphs In this paper, we will be working with two curve graphs, $C\left(S_{g, 1} ; h\right)$ and $C\left(S_{g} ; h\right)$. The graph $C\left(S_{g, 1} ; h\right)$ has vertices corresponding to oriented isotopy classes of simple closed curves in the primitive homology class $h \in H_{1}\left(S_{g, 1} ; \mathbb{Z}\right)$. Any two vertices representing disjoint curves whose difference bounds one or more subsurfaces of $S_{g, 1}$ of genus one are connected by an edge. The graph $C\left(S_{g}, h\right)$ is defined analogously.

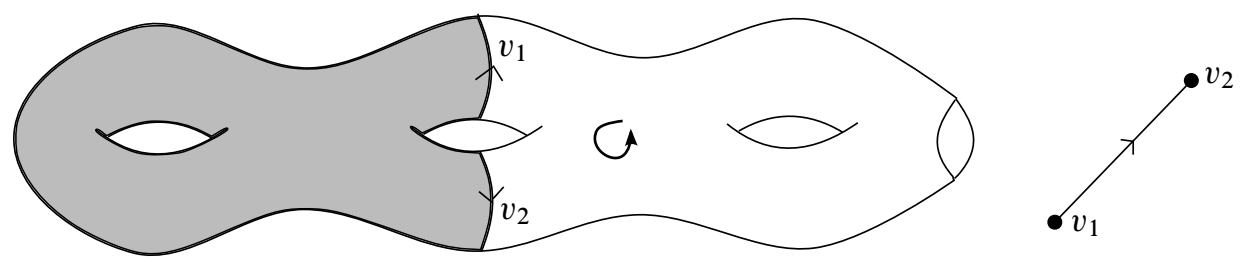

Figure 1: The shaded subsurface represents an edge of the curve graph $C\left(S_{3,1}, h\right)$.

Since there are no bounding pairs in $S_{2}$, the graph $C\left(S_{2} ; h\right)$ does not have any edges. For this reason, and also because the Johnson homomorphism, and hence the Chillingworth class, is zero in genus two (see Johnson [10, Lemma 4A]), we will not be considering surfaces of genus two.

Signed distance There is of course the usual combinatorial distance $d\left(v_{1}, v_{2}\right)$ defined on the graphs $C\left(S_{g, 1} ; h\right)$ and $C\left(S_{g} ; h\right)$. Since a path in $C\left(S_{g, 1} ; h\right)$ or $C\left(S_{g} ; h\right)$ defines a surface in a 3-manifold, as described in Irmer [9], the combinatorial distance is a special case of the Thurston norm defined in [15]. To turn this norm into a cohomology class, orientation information in the form of a signed distance $d_{s}\left(v_{1}, v_{2}\right)$ on $C\left(S_{g, 1} ; h\right)$ or $C\left(S_{g} ; h\right)$ is utilized.

Calculating the Thurston norm, or the very closely related Gromov norm, involves finding an infimum analogous to the infimum that is a stable length of a group action on a metric space such as a graph. Let $\eta$ be an endomorphism of a free group $F$. In [3, Section 9] the Gromov norm of the HNN-extension of $F$, denoted $F_{* \eta}$, was shown to be a "translation length" of $\eta$ acting on $K(F, 1)$. The "translation length" in this context was defined to be a stable commutator length, whereas translation length (also called stable length) on $C\left(S_{g, 1}, h\right)$ is a commutator length. The action of an element of the mapping class group of $S_{g, 1}$ on the fundamental group of the surface gives an endomorphism of a free group. Calegari and Walker [3] emphasized that the Chillingworth class is a rotation quasi-morphism. Rotation quasi-morphisms, defined in Calegari [2, Section 2.2], are used to estimate, or sometimes calculate, stable commutator length. 
Recall the convention that the boundary of a subsurface of $S_{g, 1}$ or $S_{g}$ is oriented in such a way that it has the subsurface to its left. A subsurface of $S_{g, 1}$ or $S_{g}$ is assigned +1 if it is oriented as a subsurface of $S_{g, 1}$ or $S_{g}$, and -1 otherwise. In particular, this gives an orientation to the edges of the curve graphs, as shown in Figure 1.

Choose a path $\gamma$ connecting $v_{1}$ and $v_{2}$ in $C\left(S_{g, 1} ; h\right)$ or $C\left(S_{g} ; h\right)$, and let $l_{s}(\gamma)$ be the number of edges traversed by $\gamma$ in the positive direction minus the number of edges traversed in the negative direction. In Lemma 3 it is shown that $l_{s}(\gamma)$ is independent of the choice of $\gamma$ in $C\left(S_{g, 1}, h\right)$. In this case, the signed distance $d_{s}\left(v_{1}, v_{2}\right)$ is defined to be $l_{s}(\gamma)$. In Lemma 2, it is also shown that for $S_{g}$ the signed length of $\gamma$ is only independent $\bmod (g-1)$ of the choice of $\gamma$. The signed distance is defined to be the equivalence class $\bmod (g-1)$. The surface $S_{3}$ is a special case, because as shown in Figure 2, a bounding pair cuts $S_{3}$ into two subsurfaces of genus one, so it is not clear how to orient edges of the curve graph. However, +1 is congruent to $-1 \bmod (3-1)$ so any arbitrary choice of edge orientation will determine the same signed length of paths $\bmod 2$.

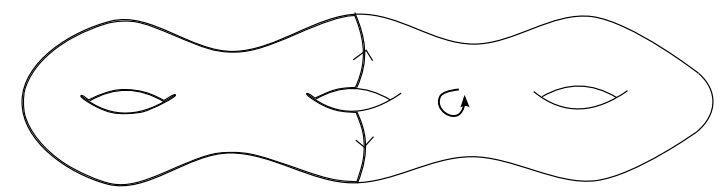

Figure 2: A bounding pair cuts $S_{3}$ into two surfaces of genus 1 .

Since the respective Torelli groups preserve orientation and act on $C\left(S_{g}, h\right)$ and $C\left(S_{g, 1}, h\right)$ by isometry, signed distance is also preserved by the Torelli group.

Signed stable length Choose a primitive homology class $h$ and define the signed stable length $\phi_{h}(\tau)$ of an element $\tau$ of $\mathcal{T}_{g, 1}$ acting on $C\left(S_{g, 1} ; h\right)$ to be

$$
\phi_{h}(\tau)=\lim _{n \rightarrow \infty} \frac{d_{s}\left(v, \tau^{n} v\right)}{n},
$$

where $v$ is any vertex of $C\left(S_{g, 1} ; h\right)$. It is not a priori clear that this is well-defined, however this will be shown to follow from Lemma 2.

By a construction due to Hatcher [8], $C\left(S_{g, 1} ; h\right)$ and $C\left(S_{g} ; h\right)$ are seen to be connected. Since the respective Torelli groups act on the curve graphs $C\left(S_{g, 1} ; h\right)$ and $C\left(S_{g} ; h\right)$ by isometry, it follows immediately that stable length, signed or otherwise, is locally independent of the choice of vertex $v$, and hence globally independent by connectivity. Lemma 4 states that $\phi_{h}(\tau)=d_{s}(v, \tau v)$ for any vertex $v$ in $C\left(S_{g, 1} ; h\right)$. For surfaces without boundary we have to make sense of what it means to stabilize a quantity that is 
only defined $\bmod (g-1)$. In analogy with surfaces with boundary, for $\tau \in \mathcal{T}_{g}$ and $h$ a primitive element of $H_{1}\left(S_{g} ; \mathbb{Z}\right)$ we define $\phi_{h}(\tau):=d_{s}(v, \tau v)$. For both $C\left(S_{g, 1} ; h\right)$ and $C\left(S_{g} ; h\right)$, if $h$ is not primitive, ie $h=\lambda[c]$ for a primitive curve $c$, then we define $\phi_{h}(\tau):=\lambda \phi_{[c]}(\tau)$.

Observe that, unlike for the mapping class group acting on the curve complex, a different stable length is obtained depending on the choice of homology class defining the graph on which the element of the Torelli group acts. An illustrative example is given by the action of a bounding pair map $T_{a} T_{b}^{-1}$. Here $T_{a}$ denotes a Dehn twist around the curve $a$, and it is assumed that the oriented multicurve $a-b$ is the boundary of a subsurface of genus 1 . Since there are curves left invariant under the action of $T_{a} T_{b}^{-1}$, its orbit in Harvey's complex of curves stays within a constant distance of vertices representing these invariant curves, hence the action of $T_{a} T_{b}^{-1}$ on Harvey's complex of curves has stable length zero. The calculation in Section 3.4 gives a formula for signed stable length of $T_{a} T_{b}^{-1}$ on $C\left(S_{g, 1}, h\right)$, depending on the algebraic intersection number of $[a]$ with $h$. For bounding pair maps, arguing as in Section 3.4, it is not hard to show that signed stable length is equal to unsigned stable length on $C\left(S_{g, 1}, h\right)$.

It is finally possible to state the theorem of this paper.

Theorem 1 The signed stable length $\phi$ is half the Chillingworth class.

Acknowledgements Thanks to Andrew Putman and Dan Margalit for discussions on earlier incarnations of this result, and to Danny Calegari, Allen Hatcher and a very thorough reviewer for comments and improvements. This work was funded by a MOE AcRF-Tier 2 WBS grant number R-146-000-143-112.

\section{Some background on the Chillingworth class}

In this section, suppose all curves are closed, simple, and oriented, with continuously varying, nowhere zero tangent vector. Let $X$ be a nowhere zero vector field on $S_{g, 1}$. In [4], Chillingworth defined the winding number of a curve $\gamma$ with respect to $X$. When presented precisely and in all generality, the definition is quite long, so only the general idea of the special case needed for studying the mapping class group will be given here.

Intuitively, the winding number $\omega_{X}(\gamma)$ is the number of times the tangent vector to $\gamma$ rotates relative to $X$ as $\gamma$ is traversed once in its positive direction. To relate winding numbers to cohomology classes, it was shown that $\omega_{X_{1}}(\gamma)-\omega_{X_{2}}(\gamma)$ only depends on the integral homology class of $\gamma$. 
In [5] applications of winding number to the study of mapping class groups were discussed. At the end of this paper the conjecture was made that for $\tau \in \mathcal{T}_{g, 1}$, if $\omega_{\tau^{*} X}(\gamma)-\omega_{X}(\gamma)=0$ for any curve $\gamma$, then $\tau$ must be in the subgroup of the Torelli group generated by Dehn twists around separating curves. In [10], Johnson showed this conjecture to be false, and started calling the cohomology class $d\left(\tau^{*} X, X\right)$, defined by

$$
\langle d(\tau * X, X),[\gamma]\rangle=\omega_{\tau^{*} X}(\gamma)-\omega_{X}(\gamma),
$$

the Chillingworth class. This cohomology class can be shown to be independent of the choice of $X$. Johnson constructed a homomorphism whose kernel, the Johnson kernel, he showed to be the subgroup of the Torelli group generated by Dehn twists around separating curves [12].

In [4, Section 6], winding numbers on surfaces without boundary were defined. For surfaces without boundary and genus greater than or equal to two, there are no nonvanishing vector fields, so suppose $X$ only has one zero; call this point $p$. When a smooth homotopy moves the curve $\gamma$ over $p$, by the Poincaré-Hopf index theorem the winding number of $\gamma$ is changed by $\pm(2-2 g)$. It follows that for surfaces without boundary, the Chillingworth class is only defined $\bmod (2 g-2)$.

\section{Proof of Theorem 1}

Proof The proof of the theorem is broken up into several parts and occupies all of this section.

\subsection{Signed distance}

In this subsection, some basic properties of signed distance on $C\left(S_{g, 1} ; h\right)$ and $C\left(S_{g} ; h\right)$ are established.

A curve is an oriented isotopy class of closed loops not homotopic into the boundary of $S_{g, 1}$. Where there is no risk of ambiguity, the same symbol will be used for a curve and a particular representative of the isotopy class. Also, in order to show that signed length only depends on the endpoints of the path, or only on the endpoints of the path $\bmod (g-1)$, properties of surfaces in the 3-manifolds $S_{g} \times I$ and $S_{g, 1} \times I$ will be used. In both cases, the surface $S_{g}$ or $S_{g, 1}$ maps into the 3-manifold. Where this does not cause confusion, the same notation will be used for a curve $c$ on a surface (thought of as $S_{g, 1} \times 0$ or $S_{g} \times 0$ ) and a representative of the isotopy class of a map of a closed loop into the 3 -manifold that also contains $c$.

A surface invariant will now be defined that is important in the proof of the next lemma. 
The pre-image function This definition is taken from [9]. Let $H$ be a connected, immersed surface in $S_{g} \times I$, and let $\hat{i}(a, b)$ denote the algebraic intersection number, either in the 3-manifold or in a surface, depending on the context. Curve graph distances are related to surfaces via the pre-image function $p_{H}: S_{g} \times 0 \backslash \pi(\partial H) \rightarrow \mathbb{Z}$. Suppose $P:=S_{g} \times I$ and $B$ are open sets in $\left(S_{g} \times\{0\}\right) \backslash \pi(\partial H)$. The algebraic intersection number provides a map $H_{2}(P, \partial H) \times H_{1}(P, B) \rightarrow \mathbb{Z}$. For $x$ in $\left(S_{g} \times\{0\}\right) \cap B$ set

$$
p_{H, B}(x):=\hat{i}(H, x \times I) .
$$

For all $x \subset S_{g} \times 0 \backslash \pi(\partial H)$ there is a choice of $B$ such that $x \subset B$. To see that the function $p_{H, B}$ does not depend on the choice of open set $B$, note that if $B \subset B^{\prime} \subset$ $\left(S_{g} \times 0\right) \backslash \pi(\partial H)$ then it follows from the naturality of the intersection pairing with respect to inclusion [6, Proposition 1.3.4] that the diagram below commutes:

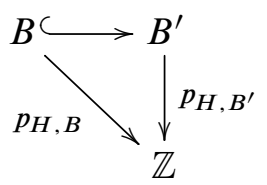

If $B_{1} \subset B_{2} \subset B_{3} \subset \cdots$ and $D_{1} \subset D_{2} \subset \cdots$ are any two sequences of open subsets of $\left(S_{g} \times\{0\}\right) \backslash \pi(\partial H)$ with direct limit $\left(S_{g} \times\{0\}\right) \backslash \pi(\partial H)$, it follows that they both give rise to the same function $p_{H}$ as the sequence $B_{1} \cap D_{1} \subset B_{2} \cap D_{2} \subset \cdots$, which also has direct limit $\left(S_{g} \times\{0\}\right) \backslash \pi(\partial H)$. Hence $p_{H}$ is well defined on $\left(S_{g} \times\{0\}\right) \backslash \pi(\partial H)$, and is extended to an upper-semicontinuous function defined on all of $S_{g} \times\{0\}$. An example is shown in Figure 3.

The pre-image function is defined analogously for $S_{g, 1}$.

Surfaces and curve graph paths Let $c_{1}$ and $c_{2}$ be curves in $S_{g, 1} \times I$ or $S_{g} \times I$ isotopic to their projections to $S_{g, 1} \times\{0\}$ or $S_{g} \times\{0\}$. These projections are assumed to be in general and minimal position and represent the vertices $v_{1}$ and $v_{2}$ respectively. Suppose $\pi$ is the projection of $S_{g} \times I$ onto $S_{g} \times\{0\}$, or of $S_{g, 1} \times I$ onto $S_{g, 1} \times\{0\}$. In [9, Section 2.1] it is explained how to construct a surface in $S_{g} \times I$ with boundary $c_{2}-c_{1}$ from an oriented path in a curve graph passing from vertex $v_{1}$ to vertex $v_{2}$. A simpler case of this is sketched below. Strictly speaking, the objects so obtained are often only cell complexes, but are homotopic to immersed surfaces with boundary.

Suppose a path in $C\left(S_{g} ; h\right)$ has $j$ edges labeled $i=1, \ldots, j$, where $j>1$. Informally, each edge of $C\left(S_{g} ; h\right)$ represents an oriented subsurface $F_{i}$ of $S_{g}$ which maps into $S_{g} \times\{i /(j+1)\} \subset S_{g} \times I$. Pairs of homotopic curves with opposite orientations on the boundaries of $F_{i}$ and $F_{i+1}$ are connected up by gluing in cylinders. Similarly, the boundary of the surface is put into the boundary of the 3-manifold by gluing on cylinders. The same construction works for $S_{g, 1} \times I$. 


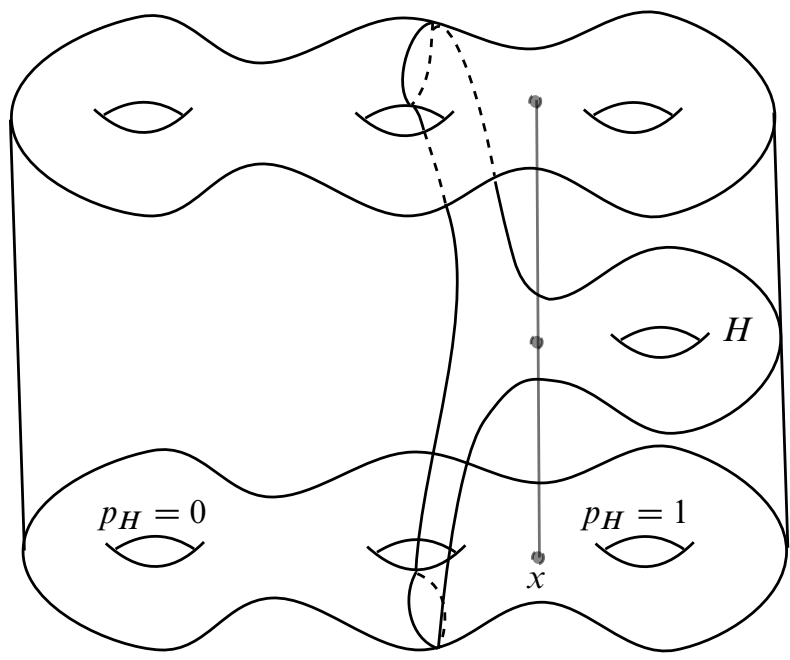

Figure 3: In this example, the pre-image function is equal to one on one side of a bounding pair, and zero elsewhere.

A surface constructed from the path $\gamma$ will be called a trace surface of $\gamma$. The trace surface of an oriented path in $C\left(S_{g} ; h\right)$ or $C\left(S_{g, 1} ; h\right)$ is unique up to homotopy.

Remark The vertices $v_{1}$ and $v_{2}$ are connected by paths of finite length. The correct intuition is that each edge of a given path represents a surface with boundary that contributes at most \pm 1 to the pre-image function on any given subsurface. It follows that the pre-image function is finite, and each level set has a finite number of connected components. A simple means of calculating the pre-image function and hence verifying finiteness is given in [9, Section 4].

Euler integrals Throughout this paper we will want to compute a "signed genus", coming from a "signed Euler characteristic". The signed genus of the trace surface of $\gamma$ is equal to the signed length of $\gamma$. To calculate signed Euler characteristic in such a way that the signs work out automatically, a very convenient notation makes use of integration with respect to Euler characteristic. The simple formulation given here is [1, Definition 3].

A collection $\mathcal{A}$ of subsets of a topological space $X$ is said to be tame if $\mathcal{A}$ is closed with respect to the operations of finite intersection, finite union and complement, and all elements of $\mathcal{A}$ possess well-defined Euler characteristics.

An indicator function $1_{U}$ is the function that is equal to 1 on the set $U$ and zero elsewhere. 
Let $\mathcal{A}$ be a tame collection and $f=\sum \lambda_{\alpha} 1_{U_{\alpha}}$ a finite $\mathbb{Z}$-linear combination of indicator functions of elements $U_{\alpha} \in \mathcal{A}$. Then the integral of $f$ is defined to be

$$
\int_{X} f d \chi:=\sum_{\alpha} \lambda_{\alpha} \chi\left(U_{\alpha}\right)
$$

Lemma 2 [16, Statement 1A] The integral in (4) is independent of the way in which $f$ is written as a linear combination of indicator functions.

Proof In this paper we can give $S_{g, 1}$ or $S_{g}$ the structure of a simplicial complex, such that all the $U_{\alpha}$ can be chosen to be closed simplices of the simplicial complex. It then follows from a simple argument (see [1, Lemma 4]) that the integral is independent of the way $f$ is written as a finite sum of indicator functions. These assumptions are, however, not necessary, and the lemma follows from the sheaf-theoretic approach in [14].

Remark Pre-image functions were defined to be upper-semicontinuous because we will want to relate their Euler integrals to signed genus of trace surfaces. Although the boundary of a trace surface has Euler characteristic zero, its projection to $S_{g} \times\{0\}$ or $S_{g, 1} \times\{0\}$ does not, so it is necessary to be careful what value to assign it. Upper semicontinuity ensures that a union of surfaces gives rise to a pre-image function that is the sum of the pre-image functions of the surfaces.

Lemma 3 Let $v_{1}$ and $v_{2}$ be two vertices of $C\left(S_{g, 1} ; h\right)$. All paths in $C\left(S_{g, 1} ; h\right)$ connecting $v_{1}$ to $v_{2}$ have the same signed length. In $C\left(S_{g} ; h\right)$, all paths connecting $v_{1}$ to $v_{2}$ have the same signed length $\bmod (g-1)$.

Proof By [9, Lemma 10] any surface in $S_{g} \times I$ with boundary $c_{2}-c_{1}$ has the same pre-image function up to an additive constant. The same argument applies to $S_{g, 1} \times I$, although it is not explicitly stated.

We first prove the statement for surfaces with boundary, and then explain why surfaces without boundary are different. Let $\gamma_{1}$ and $\gamma_{2}$ be paths connecting $v_{1}$ to $v_{2}$ in $C\left(S_{g, 1} ; h\right)$, where $H_{1}$ is the trace surface of $\gamma_{1}$ and $H_{2}$ the trace surface of $\gamma_{2}$. As before, $\partial H_{1}=\partial H_{2}=c_{2}-c_{1}$. In this context, $c_{2}$ is a curve in $S_{g, 1} \times\{1\}$ and $c_{1}$ is a curve in $S_{g, 1} \times\{0\}$. Both curves are identified with vertices in the curve graph by projecting isotopy classes of $S_{g, 1} \times I$ onto isotopy classes of $S_{g, 1} \times\{0\}$. By assumption, neither $\pi c_{2}$ nor $c_{1}$ is homotopic to $\partial S_{g, 1} \times\{0\}$. From the definition of pre-image function, it follows that $p_{H}$ must be zero on some neighborhood of $\partial S_{g, 1}$. Since $p_{H_{1}}-p_{H_{2}}$ is a constant, it follows that $p_{H_{1}}=p_{H_{2}}$. 
Recall that the surface $H_{1}$ is homotopic to a union of genus one subsurfaces $F_{i}$ connected along boundary curves. Each of the $F_{i}$ projects one to one onto an embedded, incompressible subsurface of $S_{g, 1} \times 0$ of genus 1 . In order to define the pre-image function of $F_{i}$ for some $i$, strictly speaking the boundary of $F_{i}$ should be put in the boundary of the 3-manifold. This detail will be ignored, because it is not hard to see that a homotopy taking $F_{i}$ to $F_{i}^{\prime}$ can be found, such that $p_{F_{i}^{\prime}}$ is one on the interior of the subsurface $\pi\left(F_{i}\right)$ of $S_{g}$ or $S_{g, 1}$ and zero on $S_{g} \backslash \pi\left(F_{i}\right)$ or $S_{g, 1} \backslash \pi\left(F_{i}\right)$. The pre-image function $p_{H}$ is the sum of the pre-image functions of the $F_{i}$, except along the curves where annuli are glued in to join the $F_{i}$ together, over which $p_{H}$ varies continuously.

The signed genus of $F_{i}$ is obtained by

$$
-\frac{1}{2} \int_{S_{g}} p_{F_{i}} d \chi
$$

Depending on the orientation of $F_{i}$, this will be plus or minus one. Since integrals preserve finite linear combinations, it follows that the signed length of $\gamma_{1}$ is equal to

$$
-\frac{1}{2} \int_{S_{g, 1}} p_{H_{1}} d \chi
$$

which is independent of the choice of $\gamma_{1}$ because $p_{H_{1}}$ is.

The proof is the same for surfaces without boundary, except that paths can be chosen such that the pre-image functions of the trace surfaces differ by any integer. To see this, we show how to increase or decrease the pre-image function by one. Suppose $v$ is a vertex on $\gamma_{1}$ corresponding to the curve $c$. Cut $H_{1}$ along the curve $c$, and glue in a copy of a surface homotopic in $S_{g} \times I$ to $\left(S_{g} \times\left\{\frac{1}{2}\right\}\right) \backslash\left(c \times\left\{\frac{1}{2}\right\}\right)$. The orientation of the surface glued in will determine whether the pre-image function increases or decreases by one.

To see that we need to take $\bmod (g-1)$ instead of $\bmod g$, note that when $S_{g}$ is cut into two pieces by a pair of disjoint, homologous curves, the genus of these two pieces sums to $g-1$, not $g$, as illustrated in Figure 2.

Remark The ambiguity in signed distance on $C\left(S_{g} ; h\right)$ will be related to the observation that Chillingworth's winding numbers are only defined mod $(2 g-2)$ for $S_{g}$.

When defining signed stable length on $C\left(S_{g}, h\right)$, it is necessary to make sense of the limit. If the limit exists at all, is it independent of the choice of path $\bmod (g-1)$ ? The next lemma will be used to resolve these problems. 
Lemma 4 Let $v$ and $w$ be any two vertices of $C\left(S_{g}, h\right)$, and $\tau$ an element of $\mathcal{T}_{g}$. Then for any $n \in \mathbb{N}$,

$$
d_{S}\left(w, \tau^{n} w\right) \equiv d_{S}\left(v, \tau^{n} v\right) \equiv n d_{S}(v, \tau v) \quad \bmod (g-1) .
$$

Similarly, when $w$ and $v$ are vertices of $C\left(S_{g, 1} ; h\right)$ and $\tau \in \mathcal{T}_{g, 1}$,

$$
d_{s}\left(w, \tau^{n} w\right)=d_{s}\left(v, \tau^{n} v\right)=n d_{s}(v, \tau v)
$$

Proof From Lemma 3, any path connecting $w$ to $\tau^{n} w$ can be used to calculate $d_{s}\left(w, \tau^{n} w\right)$. So suppose $\gamma$ is a path connecting $w$ to $\tau^{n} w$ passing through $v$ and $\tau^{n} v$, in that order. Suppose also that the unoriented subpath of $\gamma$ connecting $\tau^{n} v$ to $\tau^{n} w$ is the image under $\tau^{n}$ of the subpath connecting $w$ to $v$. Reversing the orientation of a path changes the sign of its signed length, and the action of $\tau$ preserves signed distance, so the signed length of the subpath connecting $w$ to $v$ cancels out the signed length of the subpath connecting $\tau^{n} v$ to $\tau^{n} w$. It follows that $d_{s}\left(w, \tau^{n} w\right) \equiv d_{s}\left(v, \tau^{n} v\right)$.

To calculate $d_{s}\left(v, \tau^{n} v\right)$, we now suppose that $\gamma$ is the union of oriented subpaths $\delta$, $\tau \delta, \ldots, \tau^{n} \delta$, where $\delta$ connects $v$ to $\tau v$. Since $\tau$ preserves signed distance, the second equality follows.

The proof is identical for $S_{g, 1}$.

\subsection{Signed stable length and cohomology}

Choose an element $\tau$ from $\mathcal{T}_{g}$ or $\mathcal{T}_{g, 1}$, and consider it fixed throughout this section. A similar argument to that in the previous section is used to show that the map from $H_{1}\left(S_{g, 1} ; \mathbb{Z}\right)$ or $H_{1}\left(S_{g} ; \mathbb{Z}\right)$ into $\mathbb{Z}$ defined by $\phi$ is a homomorphism.

Lemma 5 For fixed $\tau$, the signed stable length $\phi$ defines an element of $H^{1}\left(S_{g, 1}, \mathbb{Z}\right)$ or $H^{1}\left(S_{g}, \mathbb{Z} /\langle g-1\rangle\right)$.

Proof This lemma needs to show that $\phi$ is a homomorphism on homology. Let $\left(\left[\alpha_{1}\right], \ldots,\left[\alpha_{g}\right],\left[\beta_{1}\right], \ldots,\left[\beta_{g}\right]\right)$ be a set of generators for $H_{1}\left(S_{g, 1}, \mathbb{Z}\right)$ or $H_{1}\left(S_{g}, \mathbb{Z}\right)$. A union of oriented curves consisting of $a_{1}$ curves in the homotopy class $\alpha_{1}, a_{2}$ curves in the homotopy class $\alpha_{2}$, etc will be denoted by $a_{1} \alpha_{1}+a_{2} \alpha_{2}+\cdots$, where a minus sign denotes reversed orientation. Suppose $c$ is a curve representing the homology class $[c]$, where

$$
[c]=\sum_{i=1}^{g} a_{i}\left[\alpha_{i}\right]+b_{i}\left[\beta_{i}\right] .
$$


For any $n$, a cell complex homotopic to an immersed surface in $S_{g} \times I$ or $S_{g, 1} \times I$ with boundary $\tau c-c$ can be constructed by taking a surface $H$ with boundary

$$
\tau\left(\sum_{i=1}^{g} a_{i} \alpha_{i}+\sum_{i=1}^{g} b_{i} \beta_{i}\right)-\sum_{i=1}^{g} a_{i} \alpha_{i}-\sum_{i=1}^{g} b_{i} \beta_{i}
$$

and attaching two surfaces $F_{1}$ and $F_{2}$ with boundaries $\tau c-\tau\left(\sum_{i=1}^{g} a_{i} \alpha_{i}+\sum_{i=1}^{g} b_{i} \beta_{i}\right)$ and $\sum_{i=1}^{g} a_{i} \alpha_{i}+\sum_{i=1}^{g} b_{i} \beta_{i}-c$ respectively.

Since $\partial F_{1}$ is $-\tau\left(\partial F_{2}\right)$, the contribution of $F_{1}$ and $F_{2}$ to the signed genus of a surface with boundary $\tau c-c$ cancel out, either absolutely or $\bmod (g-1)$, depending on whether the surface is in $S_{g, 1} \times I$ or $S_{g} \times I$. This implies that

$$
\phi_{[c]}(\tau)=-\frac{1}{2} \int p_{H} d \chi
$$

For $S_{g, 1} \times I$ it was shown in Lemma 3 that the pre-image function only depends on the boundary of the surface, so $H$ can be chosen to be a union of surfaces $\left\{A_{i}\right\}$ and $\left\{B_{i}\right\}$, where $A_{i}$ is a union of $a_{i}$ copies of a surface with boundary $\tau \alpha_{i}-\alpha_{i}$ and $B_{i}$ is a union of $b_{i}$ copies of a surface with boundary $\tau \beta_{i}-\beta_{i}$. It follows that

$$
p_{H}=\sum_{i=1}^{g} p_{A_{i}}+p_{B_{i}}
$$

For $S_{g, 1}$ the lemma follows from expression (5). Similarly for $S_{g}$.

\subsection{Signed stable length is a homomorphism on Torelli}

The proof that $\phi_{h}$ is a homomorphism on the Torelli group for any $h$ is very similar to the proof of Lemma 4.

Lemma 6 The map $\phi_{h}$ is a homomorphism.

Proof Choose a primitive element $h$ of $H_{1}\left(S_{g, 1}, \mathbb{Z}\right)$ and fix it for the duration of this proof. The quantity $\phi_{h}(\tau)=d_{s}(v, \tau v)$ is then a function of one variable $\tau$, because it was shown in Lemma 4 that $\phi$ does not depend on the choice of vertex $v$ in $C\left(S_{g, 1}, h\right)$. Suppose that $\tau, \tau_{1}$ and $\tau_{2} \in \mathcal{T}_{g, 1}$, where $\tau=\tau_{2} \tau_{1}$. By the path independence of signed distance,

$$
d_{S}(c, \tau c)=d_{s}\left(c, \tau_{2} \tau_{1} c\right)=d_{s}\left(c, \tau_{1} c\right)+d_{s}\left(\tau_{1} c, \tau_{2} \tau_{1} c\right) .
$$

Since the action of the Torelli group preserves signed distances, it follows that

$$
d_{s}\left(c, \tau_{1} c\right)+d_{s}\left(\tau_{1} c, \tau_{2} \tau_{1} c\right)=d_{s}\left(c, \tau_{1} c\right)+d_{s}\left(c, \tau_{2} c\right)=\phi_{h}\left(\tau_{1}\right)+\phi_{h}\left(\tau_{2}\right) .
$$

The proof is identical for $\tau_{1}$ and $\tau_{2}$ in $\mathcal{T}_{g}$. 


\subsection{Signed stable length is zero on the Johnson kernel}

Recall that the Johnson kernel is the kernel of the Johnson homomorphism, and is generated by Dehn twists around separating curves [12]. Once we have evaluated signed stable length on bounding pair maps, it would be possible to use the lantern relation to write a Dehn twist around a separating curve as a product of bounding pair maps, and show that the signed distance between any two vertices of $C\left(S_{g, 1} ; h\right)$ or $C\left(S_{g} ; h\right)$ in the same orbit of the Johnson kernel is zero. However it is also not hard to show this directly from the definition, which is what will be done in this subsection. The basic idea is that a separating curve $s$ has zero algebraic intersection number with any other curve, so Dehn twisting around $s$ essentially adds as many copies of $s$ as it does $-s$.

Let $i(a, b)$ denote the geometric intersection number of the curve $a$ with the curve $b$, ie the minimum possible number of points of intersection between two curves, one of which is isotopic to $a$ and the other to $b$.

Let $c$ be a curve representing a vertex in $C\left(S_{g, 1} ; h\right)$ or $C\left(S_{g} ; h\right)$. Up to homotopy, a curve $T_{s}(c)$ can be obtained by taking the union of $c$ with $i(c, s) / 2$ copies of the curve $s$ and $i(c, s) / 2$ copies of $-s$, and performing 1 -surgeries to resolve the points of intersection, as illustrated in Figure 4. The null-homologous union of curves

$$
T_{s} c-c-\frac{i(c, s)}{2} s+\frac{i(c, s)}{2} s
$$

is the boundary of an incompressible surface $F$ in $S_{g, 1} \times I$ or $S_{g} \times I$. The support of $p_{F}$ is contained within the union of tubular neighborhoods of $c$ and tubular neighborhoods of $i(c, s)$ disjoint curves freely homotopic to $s$. Since the union of tubular neighborhoods has genus zero, so does $F$. Since

$$
-\frac{i(c, s)}{2} s+\frac{i(c, s)}{2} s
$$

is the boundary of a surface with zero signed genus, the same is true for a surface with boundary $T_{S} c-c$.

\subsection{Evaluation on the generators}

Now that $\phi$ has been shown to be a homomorphism both on the Torelli group (Lemma 6) and on $H_{1}\left(S_{g, 1}, \mathbb{Z}\right)$ and $H_{1}\left(S_{g}, \mathbb{Z} /\langle g-1\rangle\right.$ ) (Lemma 5 ), all that remains to prove Theorem 1 is to verify it on a choice of generating sets.

The Torelli group is generated by bounding pair maps. Let $(a, b)$ be a bounding pair. Choose curves representing a basis $B$ for $H_{1}\left(S_{g, 1}, \mathbb{Z}\right)$, so that only one of them, $\alpha$, 


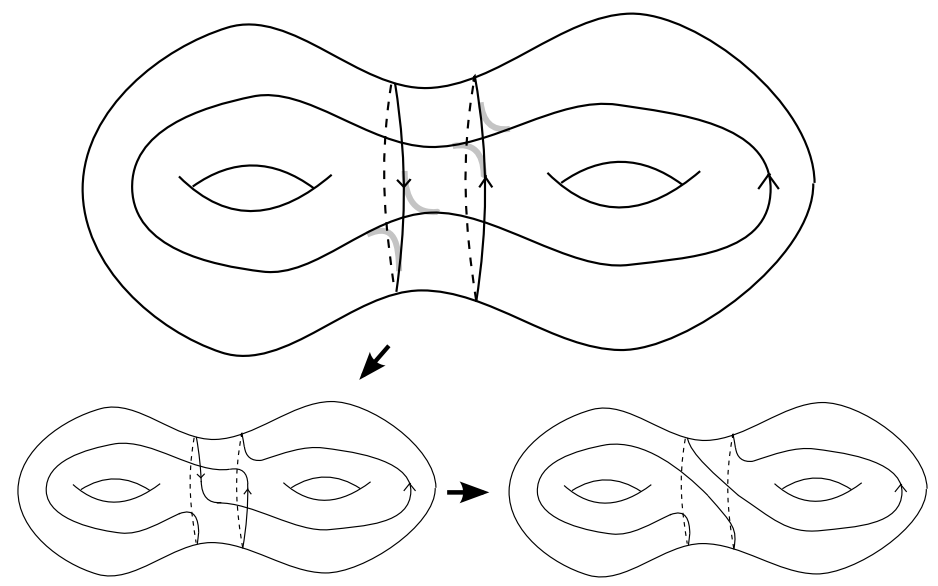

Figure 4: The surgeries to resolve the points of intersection are shown in grey in the top figure. The horizontal arrow denotes a homotopy.

intersects $a$ and $b$, and $i(a, \alpha)=i(b, \alpha)=1$. Clearly, $d_{s}\left(\beta,\left(T_{a} T_{b}^{-1}\right) \beta\right)=0$ whenever $[\beta]$ is any generator other than $[\alpha]$. The same argument as in Section 3.4 shows that $d_{s}\left(\alpha,\left(T_{a} T_{b}^{-1}\right) \alpha\right)$ is equal to the signed genus of the surface with boundary $a-b$. So $\phi_{[\alpha]}\left(T_{a} T_{b}^{-1}\right)$ is equal to the signed genus of the subsurface bounded by $a-b$ and $\phi\left(T_{a} T_{b}^{-1},[\beta]\right)=0$. This is consistent with half the Chillingworth class evaluated on this homology basis, as computed in [10, Corollary 1].

For a surface without boundary the calculation is identical. Let $B$ be a homology basis chosen as in the previous paragraph. It follows from [10, Section 6] that this is again equal to half of the Chillingworth class evaluated on the basis $B$.

This concludes the proof of Theorem 1.

\section{References}

[1] Y Baryshnikov, R Ghrist, Target enumeration over planar sensor networks, from: "Robotics: Science and Systems, IV", (O Brock, J C Trinkle, F Ramos, editors), MIT Press, Cambridge, MA (2009) 238-245

[2] D Calegari, $s c l$, Math. Soc. Japan Memoirs 20, Mathematical Society of Japan, Tokyo (2009) MR2527432

[3] D Calegari, A Walker, Surface subgroups from linear programming, Duke Math. J. 164 (2015) 933-972 MR3332895

[4] D R J Chillingworth, Winding numbers on surfaces, I, Math. Ann. 196 (1972) 218-249 MR0300304 
[5] D R J Chillingworth, Winding numbers on surfaces, II, Math. Ann. 199 (1972) 131153 MR0321091

[6] A Dold, Lectures on algebraic topology, Grundl. Math. Wissen. 200, Springer, New York (1972) MR0415602

[7] B Farb, D Margalit, A primer on mapping class groups, Princeton Math. Series 49, Princeton Univ. Press (2012) MR2850125

[8] A Hatcher, The cyclic cycle complex of a surface arXiv:0806.0326

[9] I Irmer, Geometry of the homology curve complex, J. Topol. Anal. 4 (2012) 335-359 MR2982447

[10] D Johnson, An abelian quotient of the mapping class group $\mathcal{I}_{g}$, Math. Ann. 249 (1980) 225-242 MR579103

[11] D Johnson, A survey of the Torelli group, from: "Low-dimensional topology", (S J Lomonaco, Jr, editor), Contemp. Math. 20, Amer. Math. Soc. (1983) 165-179 MR718141

[12] D Johnson, The structure of the Torelli group, II: A characterization of the group generated by twists on bounding curves, Topology 24 (1985) 113-126 MR793178

[13] S Morita, Casson's invariant for homology 3-spheres and characteristic classes of surface bundles, I, Topology 28 (1989) 305-323 MR1014464

[14] P Schapira, Operations on constructible functions, J. Pure Appl. Algebra 72 (1991) 83-93 MR1115569

[15] W P Thurston, A norm for the homology of 3-manifolds, Mem. Amer. Math. Soc. 339, Amer. Math. Soc. (1986) MR823443

[16] O Y Viro, Some integral calculus based on Euler characteristic, from: "Topology and geometry - Rohlin Seminar", (O Y Viro, editor), Lecture Notes in Math. 1346, Springer, Berlin (1988) 127-138 MR970076

Department of Mathematics, Florida State University,

208 Love Building, 1017 Academic Way, Tallahassee, FL 32306-4510, USA

iirmer@math.fsu.edu

Received: 18 December 2013 Revised: 3 September 2014 